\title{
A parametric study of the numerical simulations of triggered VLF emissions
}

\author{
D. Nunn ${ }^{1}$, M. Rycroft ${ }^{2}$, and V. Trakhtengerts ${ }^{3}$ \\ ${ }^{1}$ School of Electronics and Computer Science, Southampton University, Southampton SO17 1BJ, UK \\ ${ }^{2}$ CAESAR Consultancy, 35 Millington Rd., Cambridge CB3 9HW, UK \\ ${ }^{3}$ Institute of Applied Physics, 46 Ulyanov St., Nizhni Novgorod 603950, Russia
}

Received: 17 June 2005 - Revised: 19 October 2005 - Accepted: 20 October 2005 - Published: 23 December 2005

\begin{abstract}
This work is concerned with the numerical modelling of VLF emissions triggered in the equatorial region of the Earth's magnetosphere, using a well established 1D Vlasov Hybrid Simulation (VHS) code. Although this code reproduces observed ground based emissions well there is some uncertainty regarding the magnitude of simulation parameters such as saturation wave amplitude, cold plasma density, linear growth rate and simulation bandwidth. Concentrating on emissions triggered by pulses of VLF radio waves from the transmitter at Siple Station, Antarctica ( $\mathrm{L}=4.2$ ), these parameters, as well as triggering pulse length and amplitude, are systematically varied. This parametric study leads to an understanding of the physics of the triggering process and also of how the properties of these emissions, particularly their frequency time profile, depend upon these parameters. The main results are that weak power input tends to generate fallers, intermediate power input gives stable risers and strong growth rates give fallers, hooks or oscillating tones. The main factor determining the frequency sweep rate - of either sign - turns out to be the cold plasma density, lower densities giving larger sweep rates.
\end{abstract}

Keywords. Radio science (Waves in plasma) - Space plasma physics (Numerical simulation studies; Wave-particle interactions)

\section{Introduction}

The magnetosphere is a marvellous 3-D plasma physics laboratory in which Nature demonstrates many fascinating time varying phenomena. Using active experiments scientists investigate associated phenomena in a controlled way. A particular example where systematic investigations of wave particle interactions have complemented studies of naturally occurring phenomena (Trakhtengerts and Rycroft, 2000) is

Correspondence to: D. Nunn

(dn@ecs.soton.ac.uk) provided by investigations of whistler mode signals from the Siple radio transmitter.

Omura et al. (1991) reviewed observational, theoretical and numerical studies of VLF radio emissions triggered by the transmitter at Siple Station. The emissions are narrow band, long enduring, with an amplitude which saturates at $\sim 30 \mathrm{~dB}$ greater than the amplitude of the triggering pulse; they often sweep in frequency at a rate $\sim 1-4 \mathrm{kHz} / \mathrm{s}$. The ground based transmitters which trigger emissions are located at $2<\mathrm{L}<5$. Triggering almost always takes place within the plasmasphere rather than beyond the plasmapause. Omura et al. (1991) also explained the basic physics of whistler mode wave particle interactions, and gave expressions for the wavefield equations, the equations of motion of gyroresonant electrons and the equation for the nonlinear resonant particle current. They concluded that " numerical modelling or simulation of the VLF triggered emissions is the only way to disentangle the generation mechanism". This paper attempts to do just that.

\section{The simulation code}

\subsection{Basics of the Vlasov Hybrid Simulation method}

The simulation code for the numerical modelling of triggered VLF emissions, termed VHS/VLF, is a 1-D code which realistically assumes that the VLF waves are "ducted" through the magnetosphere. In a duct of enhanced cold plasma density the waves propagate with their wave vector approximately parallel to the ambient magnetic field. The ambient magnetic field is close to being a parabolic function of the distance $z$ from the equator. The spatial dimension of the simulation box, which straddles the equator, is fixed and is of order $6000 \mathrm{~km}$ in length (Nunn and Smith, 1996). It is in this region that significant nonlinear wave particle interaction occurs; these interactions are responsible for the triggered emissions. 
Since triggered emissions (TEs) are very narrow band $(\sim 50 \mathrm{~Hz})$, the simulation is band limited. The code bandwidth needs to be of the order of several (usually about three) trapping frequencies, in order to describe fully the self consistent interaction between the wave field and trapped resonant electrons. At every simulation time step the simulation wavefield is bandpass filtered to this width by Fast Fourier Transform (FFT) and inverse FFT (IFFT). As the frequency of the simulated emission rises or falls, the simulation centre frequency is moved at every time step to be situated at the centre of gravity of the simulation wavefield spectrum. Furthermore, since it is known that spatial gradients of frequency/wavenumber are a significant feature of VLF generation region (GR) structure (Nunn et al., 1997, 1999), the code permits wave number gradients to be established across the simulation zone.

The physical mechanism responsible for triggered VLF emissions is electron cyclotron resonance between the band limited VLF waves and keV radiation belt electrons (Rycroft, 1976). The unperturbed electron distribution function $F_{0}$ (Eq. 20) is modelled as a multiple bi-Maxwellian with a pitch angle anisotropy factor $\mathrm{A} \sim 2$, in order to provide the free energy for the instability. The linear electron cyclotron growth rate at the equator is a factor that is not too well known. We shall be using values in the range $30-150 \mathrm{~dB} / \mathrm{s}$ (Nunn et al., 2003).

Since VLF emission triggering has to be a nonlinear process, it follows that the fundamental process involved is nonlinear electron cyclotron resonance, which is synonymous with the so called phase trapping mechanism (Nunn, 1974). Trapping can only take place in the equatorial region where the ambient geomagnetic field gradients are not too large and, since the electron cyclotron resonance energy increases quickly with distance away from the equator, it is clear that emission generation takes place in this equatorial region (Nunn, 1993). It was shown by Nunn (1974) that in a parabolic magnetic field geometry a minimum wavefield amplitude ( $\sim 2 \mathrm{pT}$ in the case of Siple triggered emissions (TEs)) is necessary for nonlinear trapping to take place. Below this value the wave particle interaction behaviour is quite linear. The code itself makes no a priori assumption about trapping, nor does it require it. When run at low wave amplitudes it merely reproduces linear amplification and so does not produce triggered emissions. In this wave particle interaction (wpi) problem the resonant particle dynamics does of course depend on pitch angle, i.e. on the perpendicular velocity $\mathrm{V}_{\perp}$. However, this dependence is relatively weak. It is found that the most effective pitch angles for interaction are in the range of 45-60 deg (Nunn, 1974), since at low pitch angles the interaction tends to be linear, and since there are relatively few particles at very high pitch angles. In the code the coordinate $\mathrm{V}_{\perp}$ may be coarsely quantised - in fact here we employ 3 values. Employing a fine resolution in perpendicular velocity gives results that are little different.

The importance of inhomogeneity of the medium in the nonlinear wave particle process cannot be emphasised enough. The problem is totally different from the homogeneous case (Nunn, 1974). In an inhomogeneous medium charged particles are trapped at the now varying resonance velocity and are oscillating about a stable phase locking angle. Trapped particles now undergo a linear change of energy and magnetic moment, which results in a large value of $\mathrm{dF}$ (the perturbation of the resonant particle distribution function) in the region of phase space occupied by the "trap". This causes a large nonlinear resonant particle current whose phase corresponds to the phase locking angle (possibly $+\pi$ ). The implications for the wavefield modification are that the growth rates are much larger than the linear value due to the component of resonant particle current parallel to the wave electric field. Also there is direct modification of the wave phase due to the component of resonant particle current parallel to the wave magnetic field; this is the root cause of the sweeping frequency of emissions.

Another noteworthy feature of nonlinear wave particle interactions in an inhomogeneous medium is that the nonlinear growth rates are not only larger than the linear ones but that, unlike the homogeneous case, there is no obvious saturation mechanism in the ducted one dimensional problem. Consequently 1-D simulations tend to exhibit an absolute instability. It is therefore necessary to introduce saturation effects in a phenomenological way; this requires the selection of a saturation amplitude level in the nonlinear regime above $\sim 2 \mathrm{pT}$. The exact mechanism that results in saturation in reality is not known, but there are two obvious candidates. The first of these refers to the fact that the actual emission generating region is a 3-D object, a narrow cylinder aligned with the Earth's magnetic field. The 3-D nonlinear resonant particle current field radiates significant energy into unducted wave modes, having large angles between the wave vector and the geomagnetic field, causing considerable loss of energy. Quantifying this process numerically is currently not possible. Secondly, an electrostatic instability is expected to arise because in the presence of trapping in an inhomogeneous medium the phase averaged distribution function may exhibit positive gradients $\mathrm{dF} / \mathrm{dV}_{z}$. The resulting electrostatic wavefield is expected to result in diffusion in the $\mathrm{z}$ direction and stabilise the ambient distribution function. This is the subject of current research.

The formulation of the basic plasma physical equations in a form suitable for numerical modelling of nonlinear electron cyclotron resonance with ducted whistler waves will be found in Nunn (1990). The numerical algorithm used, Vlasov Hybrid Simulation (VHS), is highly efficient and particularly well suited to this problem. The algorithm was first introduced in Nunn $(1990,1993)$ but it has been further upgraded by Nunn (2005). For reasons of space the algorithm will not be described in detail here. The bare essentials are as follows. The time varying phase space box and grid are defined in $z, \mathrm{~V}_{z}, \psi$ space for each value of pitch angle, where $\mathrm{V}_{z}$ is velocity parallel to the geomagnetic field, $\mathrm{z}$ is distance from the equator and $\psi$ is gyrophase. Simulation particles fill phase space with a given value of density, where a figure of $1.2 /$ phase space cell is adequate. These particles are followed forwards continuously in time. Particles leaving the phase 
box are deleted from the simulation and new particles are continuously introduced at the phase box boundaries where phase fluid is flowing in. The integrated energy change dW is calculated for each particle; applying Liouville's theorem, the distribution function $\mathrm{F}$ is then known at all the phase space points occupied by particles. In order to calculate the resonant particle current it is then only necessary to interpolate $\mathrm{F}$ from the particles to the phase space grid, using a simple interpolator. The method has many powerful advantages, as follows.

1. Like all Vlasov methods the formalism is inherently low noise and far superior to "Particle in Cell" (PIC) methods in this respect (Cheng and Knorr, 1976).

2. The population of particles is dynamic, and is always confined to particles resonant with the current wavefield.

3. The method is very stable and robust against distribution function filamentation.

4. No artificial filtering or smoothing of the distribution function needs to be introduced.

5. A simple low order interpolator may be used since the interpolated distribution function is integrated over velocity space to calculate the current; the interpolated distribution function is not used in an accumulative fashion as part of the ongoing dynamics.

It should be noted that the resonant charged particle current is computed using the normal plasma physical expression, e being the charge on an electron, $F$ the distribution function, $F_{0}$ the unperturbed value, and $\mathrm{V}$ velocity space.

$\underline{J}_{\perp}=-e \iiint\left(F-F_{0}\right) V_{\perp} d^{3} V$,

where the integral is over velocity space in the region of local cyclotron resonance. In the literature (Carlson et al., 1990) frequent reference is made to the so called "particle bunching current". The reader is warned that no such thing exists. Any selection of particles exhibits phase bunching in a non trivial situation where a wavefield is present. The only current present is that given by the above expression.

\subsection{Development of the field equation}

In order to understand fully the computational results it is helpful here to derive the one dimensional field equations integrated by the code, under the assumption of parallel propagation (Nunn, 1990).

Assuming $z$ is the coordinate along the field line measured from the equator, electron plasma frequency is given by

$\Pi(z)^{2}=N_{e}(z) e^{2} / \varepsilon_{0} m$,

where both cold plasma density $\mathrm{N}_{e}(\mathrm{z})$ and electron gyrofrequency vary parabolically with $\mathrm{z}$. Electron gyrofrequency is given by

$\Omega=e B(z) / m ; \Omega(0)=(8.8) 10^{5} / L^{3}$.
To derive the field equations we employ dimensionless units as follows

$z_{\text {unit }}=1 / \bar{k} ; \bar{k}=\Pi(0) / c ; \quad t_{\text {unit }}=1 / \bar{\omega} ; \bar{\omega}=\Omega(0) / 2$.

A dimensional electric field amplitude $\mathrm{E}^{\prime}$ is defined in terms of amplitude $\mathrm{E}(\mathrm{V} / \mathrm{m})$ by the expression

$E^{\prime}=\frac{e E \bar{k}}{m \bar{\omega}^{2}}$

and dimensional resonant particle current by

$J^{\prime}=\frac{e J \mu_{0}}{2 m \bar{\omega} \bar{k}}$.

Assuming strictly parallel propagation, ignoring terms in $\lambda / R_{E}$, where $R_{E}$ is the radius of the Earth, Maxwell's equations and the linear equations of motion of the ambient cold plasma give the following field equation in dimensionless units

$$
\begin{aligned}
& {\left[\left(\frac{\partial}{\partial t}-2 i \beta\right)\left(\frac{\partial^{2}}{\partial z^{2}}-\frac{1}{c^{2}} \frac{\partial^{2}}{\partial t^{2}}\right)-\gamma_{e}(z) \frac{\partial}{\partial t}\right] E_{\perp}} \\
& \quad=\left(\frac{\partial}{\partial t}-2 i \beta\right) \frac{\partial}{\partial t} J_{\perp}
\end{aligned}
$$

where

$E_{\perp}=E_{x}+i E_{y} ; J_{\perp}=J_{x}+i J_{y}$

and $\mathrm{J}_{x}, \mathrm{~J}_{y}$ are the components of resonant particle current. In the above dimensionless equation the factor $\beta$ is given by

$\beta=1+0.5 \chi z^{2} ; \chi=9\left(10^{-14}\right) /\left(6370 L \bar{k}^{2}\right)$

for a parabolic variation of ambient magnetic field about the equator in a dipole field configuration. The factor $\gamma_{e}$ is the ratio $\left\{\mathrm{N}_{e}(\mathrm{z}) / \mathrm{N}_{e}(0)\right\}$ and is also assumed to be a parabolic function of $z$

$\gamma_{e}=1+0.5 v z^{2} ; v=0.4 \beta$.

The dimensionless dispersion relation now becomes

$k_{0}^{2}=\frac{\gamma_{e} \omega_{0}}{\left(2 \beta-\omega_{0}\right)}+\frac{\bar{\omega}^{2} \omega_{0}^{2}}{c^{2} \bar{k}^{2}}$,

where $\omega_{0}$ and $k_{0}(\mathrm{z})$ are dimensionless frequency and wave number, and dimensionless group velocity is of course given by

$V_{g}=d \omega / d k$

For a band limited ("narrow band") wave particle interaction, with bandwidths $\sim 100 \mathrm{~Hz}$, we now define a base frequency $\omega_{0}$ and the corresponding wave number $\mathrm{k}_{0}(\mathrm{z})$ through the above dispersion relation. Of course where the frequency of the simulated emission is rising or falling then the base frequency will have to be periodically redefined. Next we define dimensionless amplitude and resonant particle phasors $\bar{R}, \bar{J}$ in which the fast phase variation is divided out

$E_{\perp}=\bar{R} e^{i \Phi} ; J_{\perp}=\bar{J} e^{i \Phi}$, 
where

$$
\Phi=\omega_{0} t-\int_{0}^{z} k_{0}\left(z^{\prime}\right) d z^{\prime}
$$

Under the narrow band assumption the field and current phasors are slowly varying in space and time, and the general field equation above reduces to the form derived in Trakhtengerts and Rycroft (2000, Eq. 7)

$$
\left(\frac{\partial}{\partial t}+V_{g} \frac{\partial}{\partial z}\right) \bar{R}=-\frac{\omega_{0} V_{g} \bar{J}}{k_{0}}-\gamma(|\bar{R}|) \bar{R},
$$

where $\gamma(|\bar{R}|) \bar{R}$ represents the nonlinear unducting loss which increases sharply above the saturation level $\mathrm{R}_{\max }$. We may now define two useful quantities, $\mathrm{J}_{R}(\mathrm{z}, \mathrm{t})$ the component of resonant particle current parallel with the local wave electric field, and $\mathrm{J}_{I}(\mathrm{z}, \mathrm{t})$ the component parallel with the local wave magnetic field.

$J_{R}=\operatorname{Real}(\bar{J} / \bar{R}) \mid \overline{R \mid}, \quad J_{I}=\operatorname{Imag}(\bar{J} / \bar{R}) \overline{|R|}$.

If we express the wave phasor as

$\bar{R}=R e^{i \phi}$

where $\mathrm{R}(\mathrm{z}, \mathrm{t})$ is wave amplitude and $\phi(\mathrm{z}, \mathrm{t})$ is wavefield additional phase, then the field equation may be expressed in the following useful form (Nunn, 1990; Trakhtengerts and Rycroft, 2000)

$$
\begin{aligned}
\left(\frac{\partial}{\partial t}+V_{g} \frac{\partial}{\partial z}\right) R & =-\frac{\omega_{0} V_{g} J_{R}}{k_{0}}-\gamma(|\bar{R}|) \bar{R} \\
\left(\frac{\partial}{\partial t}+V_{g} \frac{\partial}{\partial z}\right) \phi & =-\frac{\omega_{0} V_{g} J_{I}}{R k_{0}} .
\end{aligned}
$$

Clearly the in phase component of resonant particle current $\mathrm{J}_{R}$ amplifies wave amplitude and provides growth/power input into the local wavefield, and the out of phase current $\mathrm{J}_{I}$ modifies the local additional phase. Resonant particle trapping in an inhomogeneous medium produces a very prominent $\mathbf{J}_{I}$ field (Nunn, 1990) and it is this that causes the frequency sweeping of triggered VLF emissions and chorus, though this can only be demonstrated convincingly by a full self consistent simulation, as is done here.

\subsection{Choice of unperturbed electron distribution function}

Observations of energetic electron distribution function from satellite missions such as Cluster are very variable due to the small number of counts in a given time interval. In these simulations we shall use a multiple bi-Maxwellian form as suggested by R. Horne (private communication), which is a convenient way to represent a general distribution function. In dimensionless units

$F_{0}(\mu, W)=\sum_{k=1}^{6} \alpha_{k} \exp \left[-\frac{\mu}{T_{\perp}^{k}}-\frac{(W-\mu)}{T_{\|}^{k}}\right]$ where

$\mu=V_{\perp}^{2} / 2 \beta$

is dimensionless magnetic moment and

$W=\left(V_{\perp}^{2}+V_{z}^{2}\right) / 2$

is dimensionless energy. Here the six parallel temperatures are given in $\mathrm{eV}$ by

$T_{\|}^{k}=[25 ; 250 ; 1500 ; 7000 ; 30000 ; 100000]$

the perpendicular temperatures in $\mathrm{eV}$ by

$T_{\perp}^{k}=[25 ; 400 ; 3000 ; 15000 ; 50000 ; 200000]$

and the coefficients $\alpha_{k}$ by

$\alpha_{k}=[1722 ; 5.85 ; 0.084 ; 0.009 ; 0.0007 ; 0.000007]$.

The overall anisotropy is seen to be $\mathrm{A} \sim 2$. It should be emphasised that these simulations are not unduly sensitive to the exact form of unperturbed distribution function. The most important parameter is actually dimensionless equatorial linear growth rate $\left(\gamma_{l i n}\right)$, given by the expression

$\gamma_{l i n}=\frac{\pi^{2} \bar{k}^{3} \omega_{0} V_{g}}{k_{0}^{2} N_{e}} \int_{0}^{\infty}\left\{\left|V_{\perp}\right|^{3}\left[\frac{\partial F_{0}}{\partial W}+\frac{2}{\omega_{0}} \frac{\partial F_{0}}{\partial \mu}\right]_{V z=V r e s ; z=0} d\left|V_{\perp}\right| .(26)\right.$

The second most important property of $F_{0}$ is the functional dependence on perpendicular velocity of the expression inside the integral, evaluated at the resonance velocity $\mathrm{V}_{z}=\mathrm{V}_{\text {res }}$. This represents the contribution to linear growth rate as a function of pitch angle. This will normally be a sharply peaked function with a maximum in the range 40-60 deg, which maximum increases with anisotropy.

\section{The parametric study}

\subsection{The selection of variable parameters}

A study such as this first needs the selection of a "base case". Here we choose which parameters are the most important and devise a strategy for the systematic variation of these parameters. This study is based on the Siple active VLF experiment (Helliwell and Katsufrakis, 1974; Helliwell, 1983, 1988, 2000; Helliwell et al., 1980, 1986) in which a horizontal transmitting VLF/ELF antenna was deployed on the Antarctic Polar Plateau at Siple Station at L=4.2. Over some 15 years a systematic programme of VLF magnetospheric wave injection experiments was conducted, and triggered emissions were observed at the geomagnetically conjugate station at Roberval, Quebec, Canada. In a sense the systematic variation of the parameters of the initiating pulse transmitted by Siple mirrors what we are doing here in the domain of computer modelling. The results of these numerical experiments may in the future be usefully compared in detail with the vast Siple experimental data base. 
Our choice of "base case" corresponds to a typical Siple experiment, and we select $\mathrm{L}=4.4$, the transmitted pulse frequency $\mathrm{f}=3600 \mathrm{~Hz}$, the cold plasma density at the equator $\mathrm{N}_{e}=400 / c c$ (i.e. inside the plasmapause). The input, a ducted wave at the upstream end of the spatial simulation box, is taken to be a CW pulse of length $277 \mathrm{~ms}$ and amplitude $0.1 \mathrm{pT}$, a value consistent with Siple radiated powers. For saturation amplitude we select, somewhat arbitrarily, a value of maximum in duct wave amplitude of $\mathrm{B}_{\max } \sim 8 \mathrm{pT}$; this represents fairly strong nonlinear trapping. This choice is supported by observations on Cluster and Geotail (Nunn, 1997), which show that chorus has amplitudes corresponding to very strong nonlinear trapping. The choice of unperturbed distribution function $\mathrm{F}_{0}$ is not sensitive, and so we use a multiple (6 fold) bi Maxwellian with an average anisotropy factor $\mathrm{A} \sim 2$ as described above. In this case maximum input power due to wave particle interaction comes from the local pitch angle range $40-60 \mathrm{deg}$. Thus we may usefully employ a $\mathrm{V}_{\perp}$ grid with 3 values of pitch angle at 40,50 and 60 degrees. The spatial grid contains 2048 points, the $\mathrm{V}_{z}$ grid 40 points and the gyrophase grid 20 points. The grid in $\mathrm{V}_{z}$ is centred on the local resonance velocity and has a width equal to 3 trapping widths, plus $\mathrm{V}_{z}$ range corresponding to the simulation frequency bandwidth. The total number of simulation particles is $\sim 5$ million, and on one SG Origin 200 processor $\sim 5000$ time steps are executed in $\sim 2$ days. For the base case a simulation bandwidth of $24 \mathrm{~Hz}$ is used, corresponding to observed emission bandwidths. One parameter that is neither measured nor well known is the linear equatorial growth rate at the trigger frequency, which is directly proportional to the energetic charged particle flux. Simple calculations (Nunn, 1984) and numerical experiment show that the minimum value required for triggering is $\sim 30 \mathrm{~dB} / \mathrm{s}$, and so we select a value of $60 \mathrm{~dB} / \mathrm{s}$.

We systematically vary six parameters that are deemed to be the most significant determinants of triggered emission behaviour. The first two are related to ambient magnetospheric conditions; these are cold plasma density $\mathrm{N}_{e}(\mathrm{el} / \mathrm{cc})$ at the equator and linear equatorial growth rate $\gamma_{l i n}(\mathrm{~dB} / \mathrm{s})$. The next two, related to transmitter format, reflect the Siple experiment, and are the triggering pulse length $\mathrm{L}(\mathrm{ms})$ and triggering pulse input amplitude in pT (at the edge of the simulation box). The last two parameters, system parameters related to the operation of the code, are simulation bandwidth $(\mathrm{Hz})$ and amplitude saturation level $\mathrm{B}_{\max }(\mathrm{pT})$. A number of other parameters which we have chosen not to investigate are not expected to provide any insight into the physics of VLF emission triggering or have any particular relevance with the Siple experimental program. These are frequency, L shell, the exact functional form of the zero order charged particle distribution function $\mathrm{F}_{o}$, and the dependence of cold plasma density on $z$, the distance from the equator (this dependence is also taken to be parabolic). We have also not looked at the dependence of the results on the simulation box size, on the grid geometry or on the simulation time step.

Our search strategy is as follows. Since a full exploration of 6-D parameter space is impractical, we start off with the "base case". Then we sweep each variable in turn through a suitable range, or else inscribe a trajectory through parameter space (in the case of Fig. 11 and Fig. 12). For each set of parameter values the code is run. Of primary interest is whether or not a strong, long lasting triggered emission is produced. In some cases only a short "weak" emission may result. The primary characteristic of an emission of interest is its frequency sweep rate, and indeed a lack of understanding of what controlled sweep rate was one of the motivators for this research. Since emissions triggered by the code usually are very stable for the duration of the emission, the most convenient way to present the results is as a plot of sweep rate against the parameter in question. In reality sometimes upward or downward hooks or oscillating tones may be produced. In this case we plot the sweep rates of both rising and falling parts of the emission. It should be noted that the calculated sweep rates are those at the exit of the simulation box some $4000 \mathrm{~km}$ from the equator on the side away from the transmitter. These results exclude the extra dispersion acquired when propagating down to the ground as half hop propagation, and so they cannot be directly compared with experimental observations.

\subsection{Simulation results}

\section{(a) The "base case"}

Before proceeding to the results of the parameter search it is worthwhile to present and understand the simulation results for the base case.

The base case triggers a stable riser with a sweep rate $\sim 1 \mathrm{kHz} / \mathrm{s}$, a figure common to most simulations with a cold plasma density of 400/cc (Nunn, 1974; Nunn et al., 1999). Figure 1 shows the received wave amplitude profile at the exit of the simulation box. The emission terminates naturally due to declining linear (and thus nonlinear) growth rates as the frequency rises. The steepness of the 'initial rise' of the received wave amplitude profile indicates that a strongly nonlinear process is operating and that it quickly saturates. This aspect of the simulation does not agree too well with observations which point to a more gradual attack phase (Helliwell et al., 1980). This result again points towards the occurrence of nonlinear loss processes not included in the 1-D model. The oscillations in the profile correspond to the formation of discrete sidebands with separations $\sim 50 \mathrm{~Hz}$. It was shown by Nunn (1986) that in the presence of nonlinear electron cyclotron trapping in a negative inhomogeneity (on the receiver side of the equator) then the upper sideband will be unstable, the lower sideband damped, with an optimal sideband separation being of the order of the particle trapping frequency. Here indeed the trapping frequency is $\sim 48 \mathrm{~Hz}$, so accounting for the $\sim 21 \mathrm{~ms}$ amplitude periodicity seen in Fig. 1. It is possible to regard the upward sweep in frequency as being due to the transfer of wave energy to successively higher sidebands, but such a phenomenological viewpoint does not embrace all the physics present.

Figure 2 shows the triggered riser as a frequency/time spectrogram of the output wavefield data stream. The FFT 


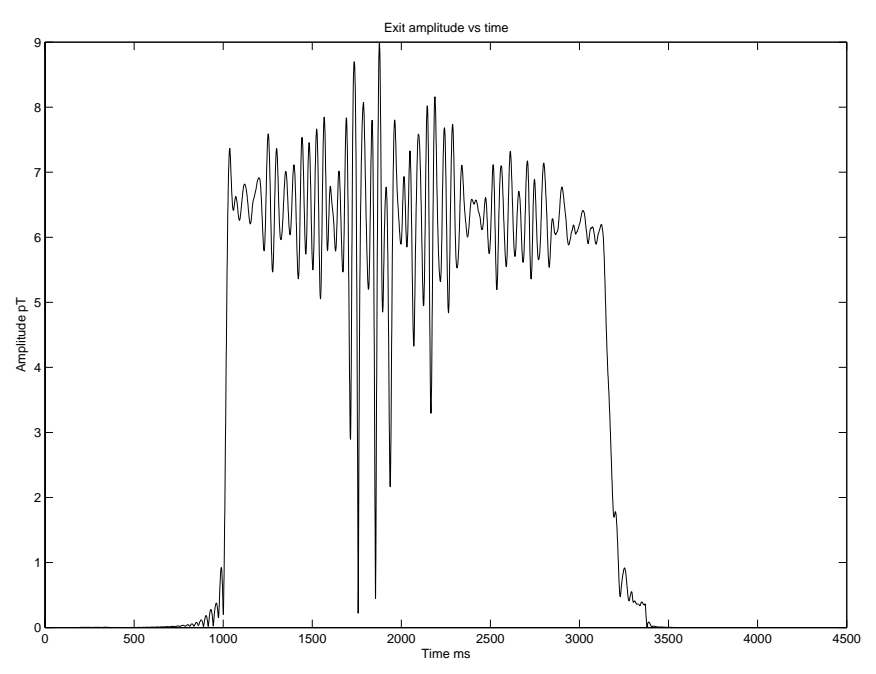

Fig. 1. Wave amplitude profile for the "base case", measured at the downstream end of the simulation box, some $4000 \mathrm{~km}$ from the equator on the receiver side. The duration of the emission is $\sim 2.2 \mathrm{~s}$. Time is measured from the start of the simulation when the triggering pulse enters the simulation box.
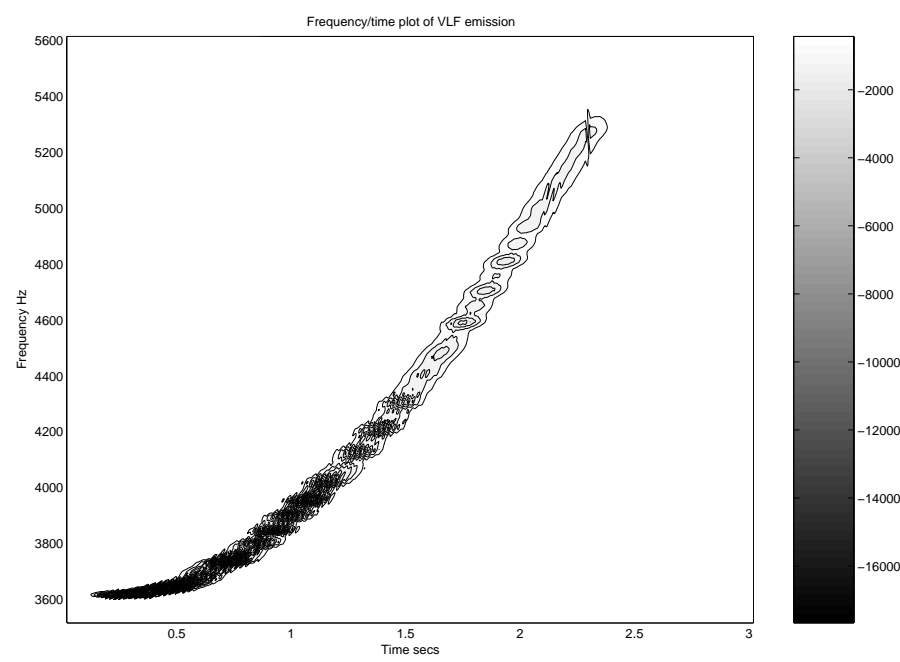

Fig. 2. Frequency-time spectrogram of the stable riser at the exit of the simulation box corresponding to the "base-case". The units of spectral power are arbitrary in all the spectrograms.

length is 1000 with a separation between successive FFT's of 250 data points.

Figure 3 shows a snapshot (at $t=1.525 \mathrm{~s}$ ) of the wavefield amplitude (in pT), the component of resonant particle current in phase with the wave electric field $\left(\mathrm{J}_{r}\right)$ and the component of resonant particle current in phase with the wave magnetic field $\left(\mathrm{J}_{i}\right)$, all as functions of $z$, the distance from the equator along the field line, positive $z$ being towards the receiver. This represents a view of the structure of the so called generating region (GR) of a triggered rising VLF emission. The characteristic structure has been noted before (Nunn et al., 1999) and is the same for all simulated risers. The GR
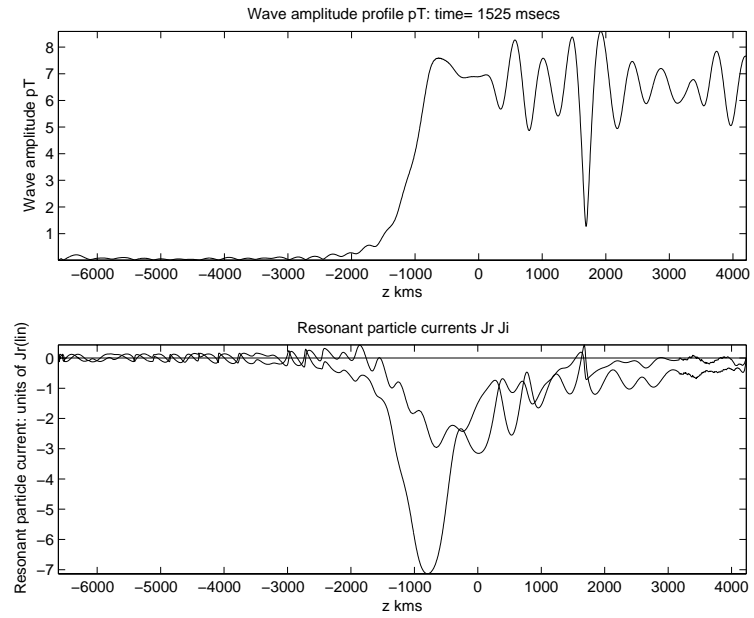

Fig. 3. Snapshot of the riser generation region in the equatorial zone at $\mathrm{L}=4.4$ for the base case at $\mathrm{t}=1525 \mathrm{~ms}$.

has a length of several thousands of $\mathrm{km}$ and is not located at a point. The GR is in the trapping region downstream of the equator (positive $z$ ). In both experimental reality and in the numerical simulations this nonlinear "soliton-like" structure is remarkably stable, and maintains its frequency sweep rate at an almost constant value. The in phase current $\mathrm{J}_{r}$ provides the power input to the wave field necessary to maintain the wave profile in a constant position. The out ${ }_{5000}$ of phase current $\mathrm{J}_{i}$ continuously modifies the wave phase in such a way as to cause the sweeping frequency. An 1000 important aspect of the mechanism whereby the frequency sweeps is that $\mathrm{J}_{i}$ generates a wave number gradient across the generation region which is then sustained. An observer -2000 at a point or fixed $\mathrm{z}$ would see frequency rise because of advection of higher frequencies from upstream. This point is discussed in more detail in Nunn (1990). Inspection of the current profiles shows that they are in good accord with concepts of trapping in a parabolic inhomogeneity. Following particles trapped by the wave from right to left the resonant particle currrent is antiparallel to the wave electric field at the commencement of trapping and rotates in phase to be parallel to the wave magnetic field at the equator where the spatial inhomogeneity of the geomagnetic field is zero. This gives a negative bell shaped profile for both components. The wave amplitude of $8 \mathrm{pT}$ gives a strongly nonlinear situation, with many trapping lengths fitting into the GR.

\section{(b) A weak emission}

Figure 4 is a frequency time spectrogram of a "weak" emission, produced by a one second duration triggering pulse with the linear growth rate reduced to $30 \mathrm{~dB} / \mathrm{s}$. Here a fully self sustaining emission is never achieved; there is a weak feature which is the termination triggering of a short faller, a phenomenon often observed in the Siple data. Also noticeable is the offset frequency of some 


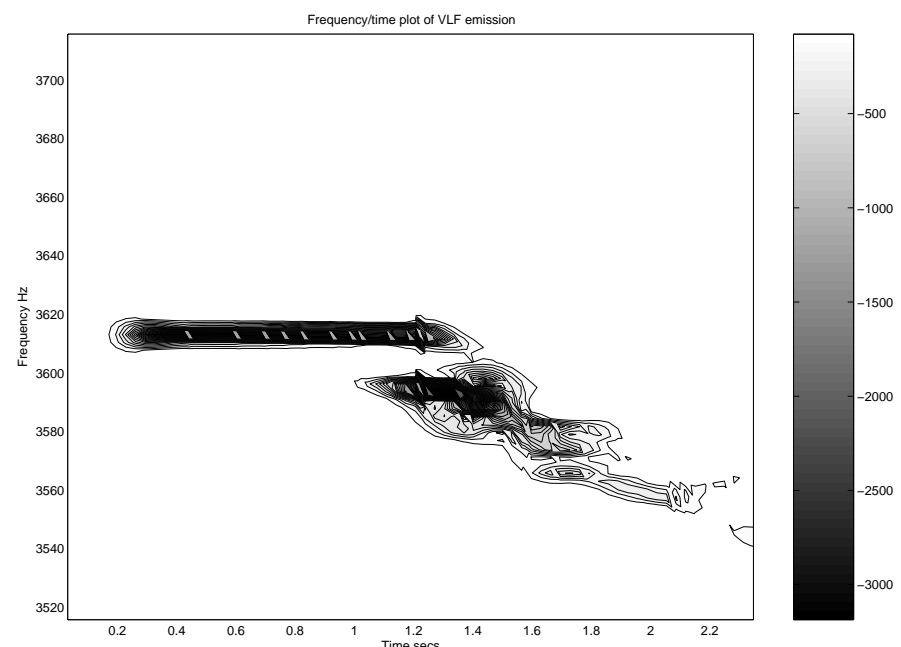

Fig. 4. Spectrogram of a weak termination faller produced by a 1 second pulse with linear equatorial growth rate of $30 \mathrm{~dB} / \mathrm{s}$. Note the pronounced offset frequency $\sim 20 \mathrm{~Hz}$.

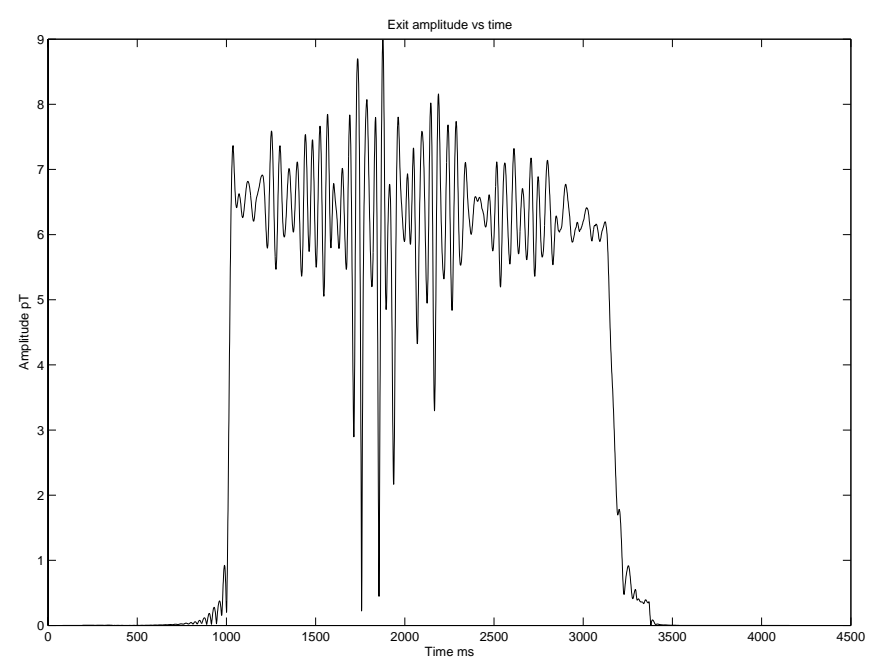

Fig. 5. Wave amplitude profile at the exit of the simulation box for the weak termination faller of Fig. 4. The strong oscillations correspond to the lower sideband wave with a separation of $\sim 25 \mathrm{~Hz}$.

$-20 \mathrm{~Hz}$, which again corresponds to the trapping frequency at the prevailing low amplitudes. Offset frequencies are also observed in the Siple data (Helliwell, 1983). The offset manifests itself as a sideband oscillation in the amplitude profile at the exit of the simulation box, as seen in Fig. 5 .

\section{(c) A falling hook emission}

Figure 6 shows the dynamic spectrogram of a triggered downward hook which consists of a short riser to $+100 \mathrm{~Hz}$ followed by a long stable faller to $-650 \mathrm{~Hz}$, with a sweep rate $\sim-400 \mathrm{~Hz} / \mathrm{s}$. This result was produced with a high linear growth rate of $90 \mathrm{~dB} / \mathrm{s}$. A positive offset $\sim+20 \mathrm{~Hz}$ is seen in the early part of the emission. Figure 7 shows a

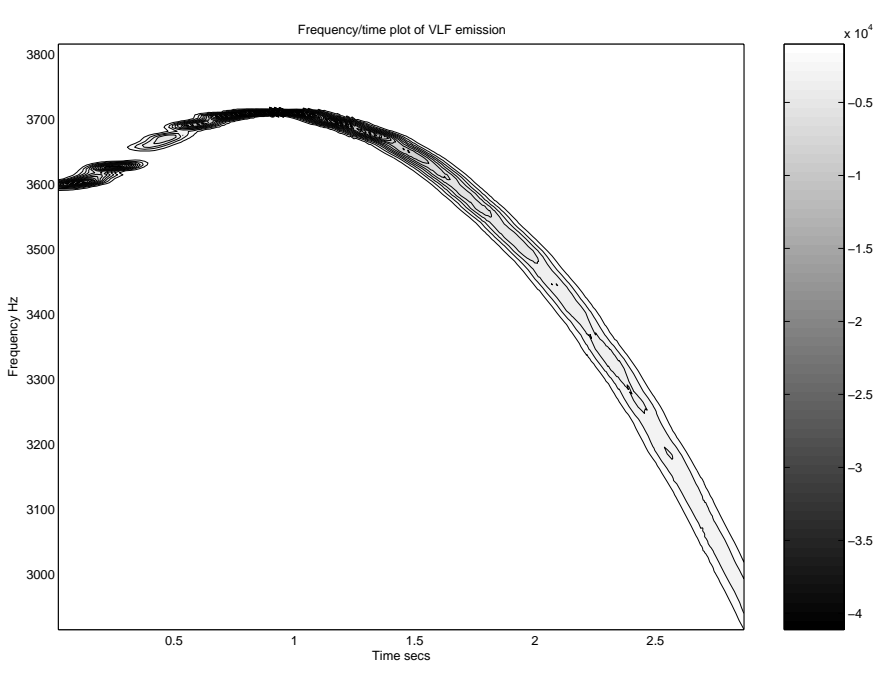

Fig. 6. Strong stable faller produced with a linear growth rate of $90 \mathrm{~dB} / \mathrm{s}$. The spectral form is actually a downward or falling hook with an offset frequency of $+20 \mathrm{~Hz}$.
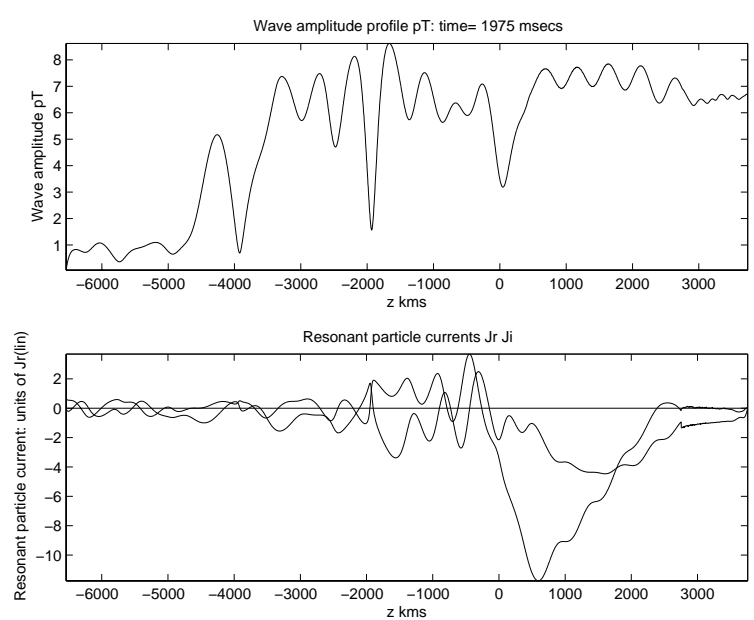

Fig. 7. Snapshot of the faller generation region of the downward falling hook shown in fig 6 , at $\mathrm{t}=1975 \mathrm{~ms}$. Note that the wave profile extends well upstream of the equator (i.e. on the transmitter side) and that consequently particle trapping occurs in this region; this results in a positive value for $\mathrm{J}_{i}$, the resonant particle current in the wave magnetic field direction. The lower curve attaining a value -12 is $\mathrm{J}_{i}$.

time snapshot of the structure of the generation region (GR) of the falling segment at time $\mathrm{t}=1.975 \mathrm{~s}$. This characteristic structure always occurs in these simulations for all strong fallers. The wave amplitude profile extends well upstream of the equator, allowing particle trapping in the positive inhomogeneity region upstream of the equator. In this region the phase locking angle rotates further towards the wave electric field giving a positive value for $\mathrm{J}_{i}$. This causes the sign of $\mathrm{df} / \mathrm{dt}$ to become negative. 


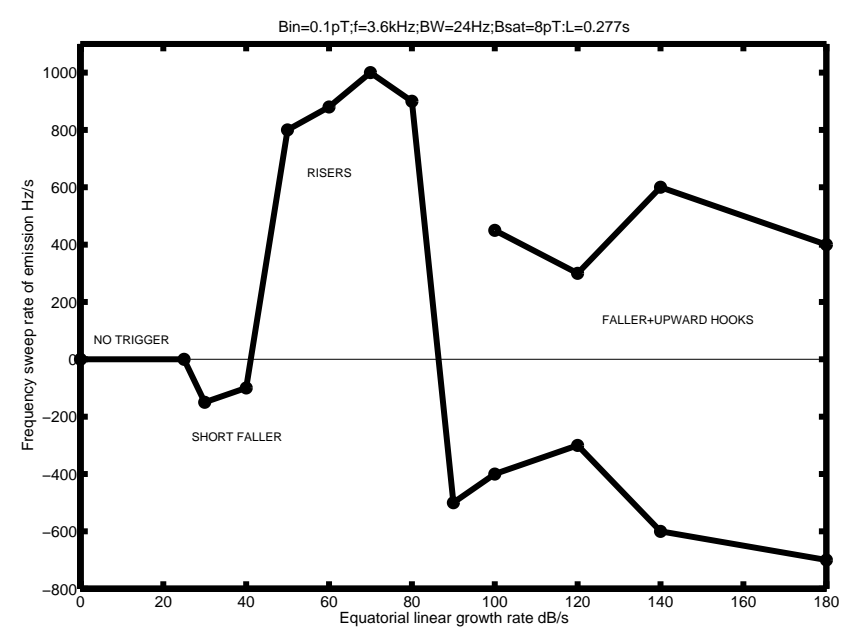

Fig. 8. Dependence of the emission sweep rate on the linear growth rate at the geomagnetic equator at $\mathrm{L}=4.4$. Note the weak fallers at low growth rates, risers at intermediate growth rates and fallers, or hooks, at high growth rates.

\section{(d) Generalisation}

Although the generation regions for risers and fallers seem to be dynamically stable, sharp transitions do occur. When the nonlinear power input to the wavefield (proportional to the linear growth rate) is increasing, as for the case of a riser for example, the wave profile can shift upstream. Then the riser GR configuration can turn into the faller configuration as trapping begins to occur upstream of the equator. This produces a downward hook. Conversely in a situation of falling power input (due to a falling linear growth rate), as for example in the case of a falling tone, where the resonance velocity is increasing, the wave profile can slip downstream. Then a faller GR becomes a riser GR, giving an upward hook. If the decrease of power input is sufficiently abrupt, a riser GR will never be established and the faller will simply terminate. In a situation where the linear growth rate is a monotonically increasing function of frequency oscillating tones may result, with a riser turning to a faller when the power input gets too high, and vice versa.

\subsection{Systematic variation of simulation parameters}

\section{(a) Variation of equatorial linear growth rate}

The first parameter to be varied is the equatorial linear growth rate at the start frequency. This is directly related to the energetic electron pitch angle anisotropy and the charged particle flux. All the other parameters remain the same as for the base case. Growth rate is swept from $25 \mathrm{~dB} / \mathrm{s}$ to $180 \mathrm{~dB} / \mathrm{s}$ and the sweep rates of the resulting triggered emissions are plotted in Fig. 8. At "intermediate" values of growth rate in the range $50-80 \mathrm{~dB} / \mathrm{s}$, a stable riser results with a sweep rate $\sim 900 \mathrm{~Hz} / \mathrm{s}$. This is a reasonable figure, in good agreement with observations and concurs with the overall tendency to trigger risers rather than fallers. At $20 \mathrm{~dB} / \mathrm{s}$ and below the growth rate is insufficient for a self sustaining GR to be

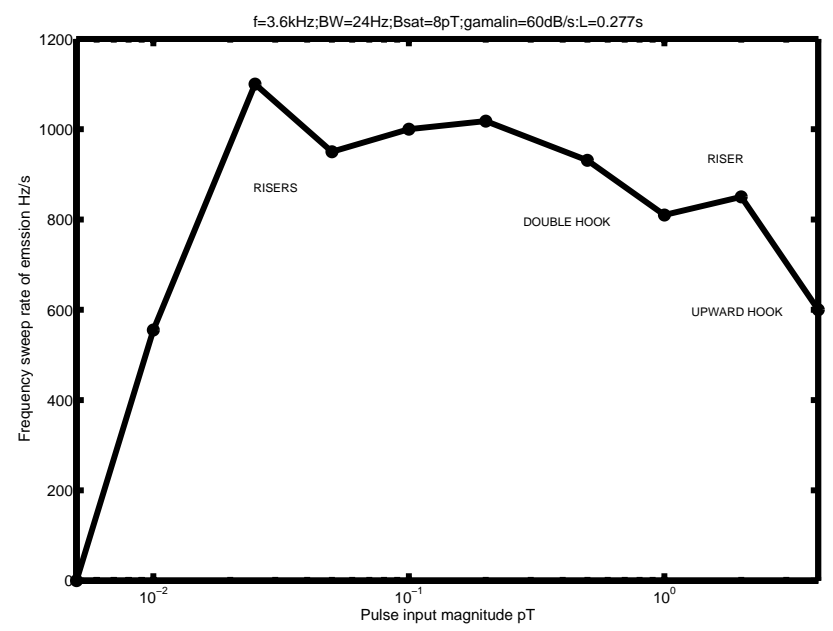

Fig. 9. Dependence of the emission sweep rate on the magnitude of the input pulse. The dependence is fairly weak; for very low amplitude input pulses triggering does not occur.

established and there is no triggering. A linear growth rate of $30 \mathrm{~dB} / \mathrm{s}$ produces a faller, but there is no long enduring self sustaining emission. At growth rates $\sim 90 \mathrm{~dB} / \mathrm{s}$ a faller GR is produced but with a sweep rate somewhat less than the corresponding riser with a value $\sim-500 \mathrm{~Hz} / \mathrm{s}$. The faller GR results from the large power input which drives the wave amplitude profile upstream. At even higher growth rates, $100-180 \mathrm{~dB} / \mathrm{s}$, these simulations produce fallers and upward hooks as expected. The two curves represent the sweep rates of both the rising and falling arms of the hooks. Interestingly they are of the same order, but have only about one half of the sweep rate of a riser on its own.

\section{(b) Variation of pulse input amplitude}

We now take the base case $\left(\mathrm{B}_{i n}=0.1 \mathrm{pT}\right)$ and vary input amplitude $\mathrm{B}_{\text {in }}$ from $0.005 \mathrm{pT}$ to $4 \mathrm{pT}$. Figure 9 plots the sweep rate of the triggered emission against input amplitude. There is a fairly consistent tendency to produce stable risers with sweep rates $\sim 1 \mathrm{kHz} / \mathrm{s}$, with only a weak dependence on input amplitude. Input amplitudes $<0.01 \mathrm{pT}$ do not trigger as the input pulse does not reach nonlinear amplitudes by the time that it reaches the equator. It thus suffers only linear amplification and propagates straight past the equator. The case of $\mathrm{B}_{i n}=0.01 \mathrm{pT}$ does succeed in triggering but with a reduced sweep rate $\sim 500 \mathrm{~Hz} / \mathrm{s}$. As the input amplitude is increased to $5 \mathrm{pT}$ (which is very unrealistic for the Siple experiment) the sweep rate slowly falls, and there is a tendency to produce hooks at very large values. Thus although $\mathrm{B}_{\text {in }}$ is a rather weak parameter it does have some effect on the final GR configuration, notably via the wave number gradient set up in the GR. The final GR configuration is, to a limited extent, then determined by the actual history of the process whereby the triggering pulse sets up the GR. The dynamics of this setup process are so complex that it is not possible to understand these results fully. 


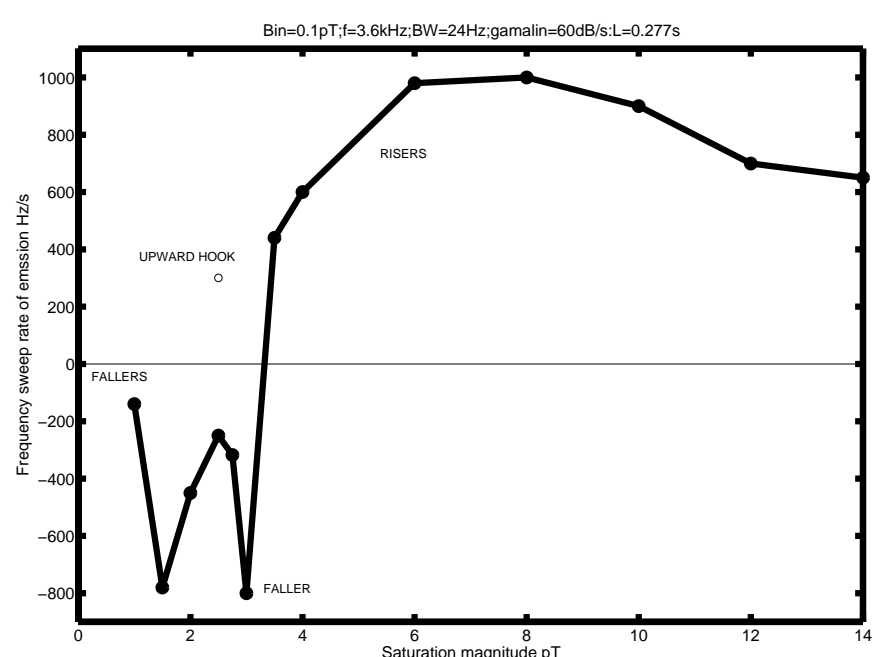

Fig. 10. Dependence of the emission sweep rate on the saturation magnitude employed in the code. Note that at magnitudes $>4 \mathrm{pT}$, well above the trapping threshold, there is little dependence. Low saturation levels are weak triggering situations, and give fallers.

\section{(c) Variation with saturation magnitude $B_{\max }$}

The next parameter to be varied is a code or system parameter, namely the saturation amplitude. Figure 10 plots the emission sweep rate against saturation magnitude, the other variables being as for the base case. At low saturation amplitudes, from 1-3 pT, trapped particles are able to execute only $\sim 1-2$ trapping oscillations in the interaction region, before they become detrapped by the increasing magnetic field gradient. The degree of nonlinearity is low and the nonlinear growth rate is of the order of the linear growth rate. It is seen that in this weak domain fallers, or in one case an upward hook, are produced. From $4 \mathrm{pT}$ to $14 \mathrm{pT}$ where the nonlinearity is strong, the triggered emissions consistently are risers with sweep rates in the region of $+900 \mathrm{~Hz} / \mathrm{s}$. The triggering behaviour is largely independent of the saturation amplitude above $4 \mathrm{pT}$, and it may seem surprising that very large wave amplitudes do not give higher sweep rates.

\section{(d) Variation with cold plasma density $N_{e}$}

The next two figures (Figs. 11 and 12) investigate the dependence of the triggered emission sweep rate on the cold plasma density $\mathrm{N}_{e}$. First we take the base case $\left(\mathrm{N}_{e}=400 / \mathrm{cc}\right)$ and vary the value of cold plasma density $\mathrm{N}_{e}$ in the equatorial plane from $50 / \mathrm{cc}$ to $800 / \mathrm{cc}$. In order to maintain the degree of nonlinear trapping about constant, we increase the saturation amplitude and the linear growth rate as $\mathrm{N}_{e}$ decreases. Strong stable risers are obtained over the whole range of cold plasma density values. Figure 11 shows the variation of emission sweep rate with $\mathrm{N}_{e}$; the sweep rate increases markedly as $\mathrm{N}_{e}$ falls. It thus is apparent that cold plasma density is the only parameter that exerts a significant control over the magnitude of sweep rate. This result is entirely in agreement with the observation of chorus elements outside the plasmapause, where ambient electron densities of

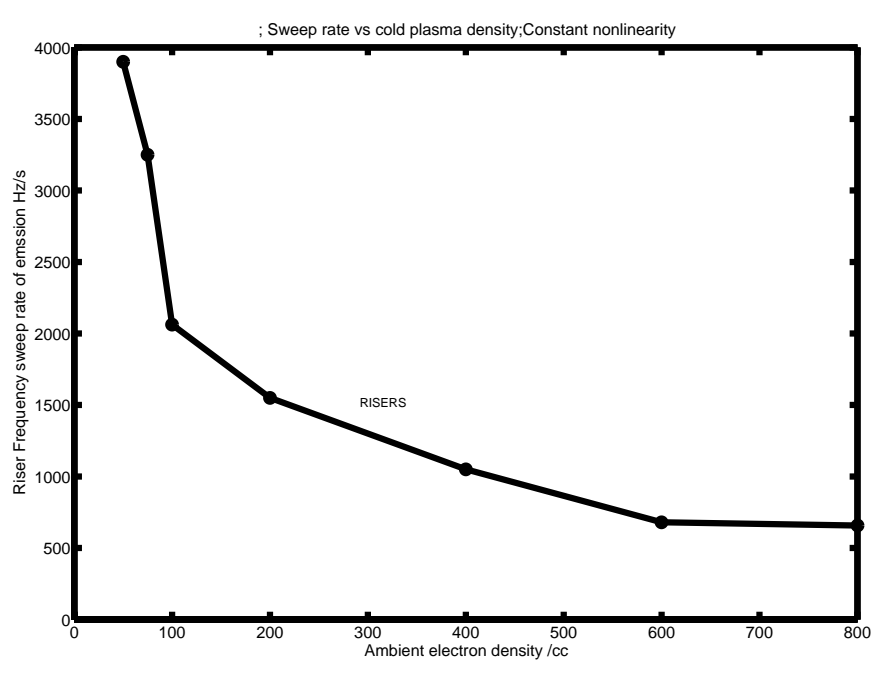

Fig. 11. Dependence of the emission sweep rate for risers on cold plasma density in the equatorial plane. The saturation amplitude and linear growth rate are simultaneously increased in the code as the density is decreased in order to maintain the same level of particle nonlinearity. Note the rapid increase of sweep rate as the density decreases. The cold plasma density is the only parameter to exert a significant control over the sweep rate of the triggered emissions.

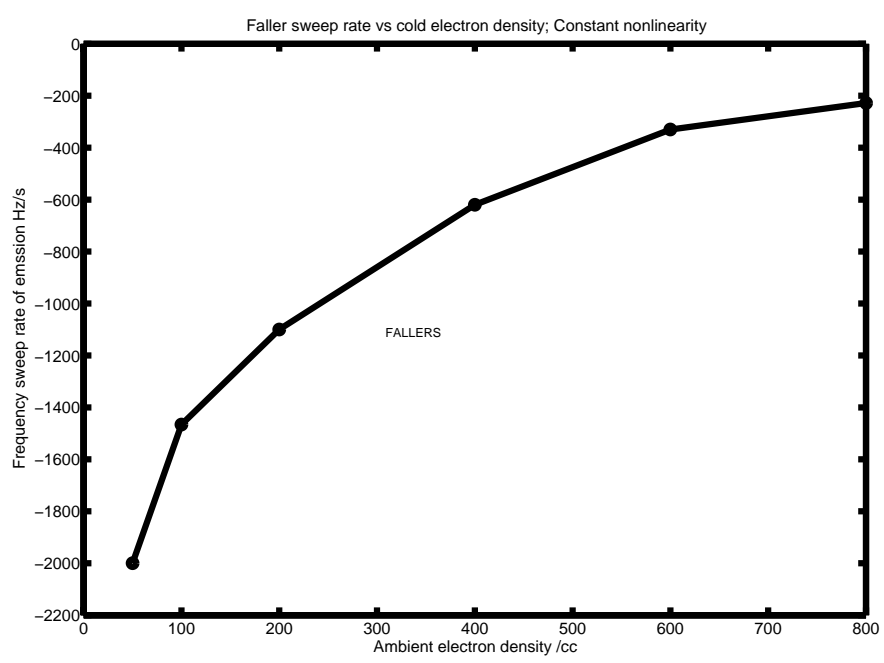

Fig. 12. Variation of the emission sweep rate for fallers as a function of cold plasma density in the equatorial plane, again at a constant nonlinearity. Again we note the strong control exerted on the sweep rate by the cold plasma density. However, the faller sweep rates are about one half of the corresponding riser sweep rate values.

only $2 / \mathrm{cc}$ are associated with sweep rates of $10 \mathrm{kHz} / \mathrm{s}$ (Santolik et al., 2003). This computational result may in the future be readily tested by correlating emission sweep rates with ambient density, either from satellite observations or from the ground, using the dispersion of whistlers to estimate the ambient density in the equatorial region. Reduced values of plasma density correspond to smaller values of the refractive index of the medium (nearer to unity). This is equivalent to 


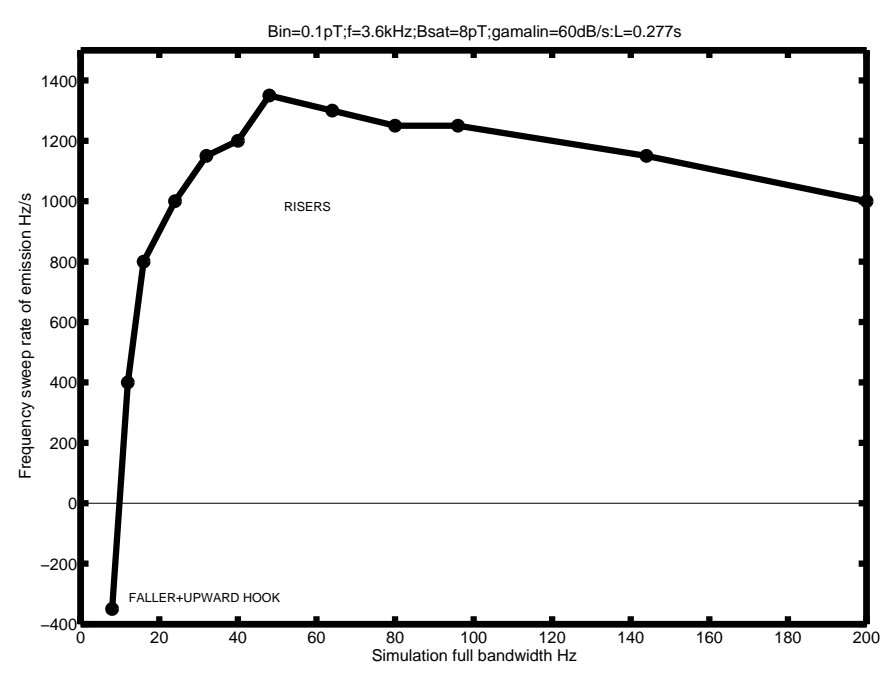

Fig. 13. Dependence of the emission sweep rate on simulation bandwidth. Above about one half the trapping frequency $(25 \mathrm{~Hz})$ there is little dependence, as the triggered emission does not occupy the whole of the available bandwidth.

gyroresonance with energetic electrons of much greater energy (Rycroft, 1976).

Figure 12 plots the corresponding graph for fallers. The initial case is the base case, but with linear growth rate equal to $90 \mathrm{~dB} / \mathrm{s}$ (a faller situation). Then again $\mathrm{N}_{e}$ is varied, with a corresponding adjustment to the linear growth rate and saturation amplitude. The result is similar to that given in Fig. 11, with the sweep rate increasing rapidly with decreasing $\mathrm{N}_{e}$. However, overall, negative sweep rates are only about half the corresponding ones for risers. It is really not surprising that $\mathrm{N}_{e}$ exerts such direct control over $\mathrm{df} / \mathrm{dt}$, as it determines the spatial scales and time scales of the whole process, and also the gyroresonance energies. Perhaps what is surprising is that the saturation amplitude and the linear growth rate seem to have almost no effect on the sweep rate.

\section{(e) Variation with simulation bandwidth}

The next parameter to be varied is simulation bandwidth, solely a code parameter. Starting with the base case bandwidth of $24 \mathrm{~Hz}$, this is swept from $8 \mathrm{~Hz}$ to $200 \mathrm{~Hz}$, and the resulting emission sweep rates are plotted in Fig. 13. For very narrow bandwidths, which are quite unrealistic, the sweep rates drop and eventually fallers result. Realistic bandwidths are $\sim>24 \mathrm{~Hz}$, of the order of the frequency of particle trapping in the potential well of the whistler mode wave, and of the order of bandwidths observed experimentally. At greater bandwidths than this, the behaviour is fairly consistent with sweep rates $\sim 1 \mathrm{kHz} / \mathrm{s}$. What tends to happen is that as the simulation bandwidth is increased the actual emission bandwidth does not expand to fill that available. The emission stays relatively narrow band. In this case expanding the simulation bandwidth will have little effect on the simulation. The conclusion is that, for realistic numerical simulations, the total bandwidth needs to be at least $20 \mathrm{~Hz}$.

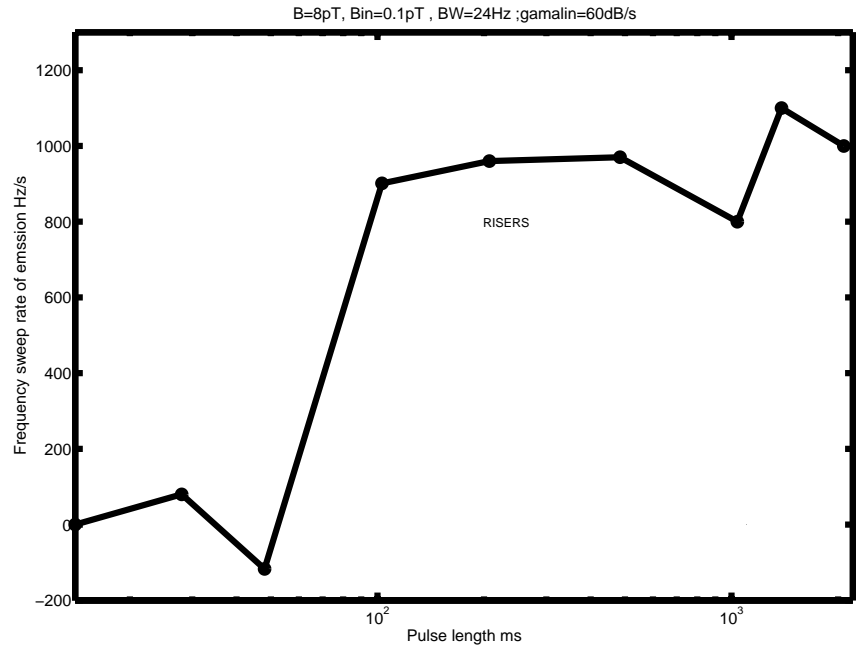

Fig. 14. Dependence of the sweep rate on the triggering pulse length. Pulses $<=50 \mathrm{~ms}$ do not produce strong self sustaining emissions as too few trapping oscillations occur within the pulse length.

\section{(f) Variation with length of triggering pulse}

The next parameter to be varied is the length of the triggering pulse. Starting with the base case $(277 \mathrm{~ms})$ pulse length is varied from $10 \mathrm{~ms}$ to $2000 \mathrm{~ms}$. The sweep rates of the emissions generated are plotted as a function of triggering pulse length in Fig. 14. At $10 \mathrm{~ms}$ no emission is produced, because the pulse is much shorter than a trapping length and particle nonlinearity does not occur. At 30 and $50 \mathrm{~ms}$ only short features are triggered with low sweep rates. This is consistent with the result that weak situations in general only produce non enduring emissions, often fallers. Above a pulse length of $100 \mathrm{~ms}$, a consistent behaviour results with long stable risers being produced with positive sweep rates $\sim 900 \mathrm{~Hz} / \mathrm{s}$ to $1000 \mathrm{~Hz} / \mathrm{s}$. For the longer pulses triggering occurs before the end of the pulse, the remainder of the initial pulse becoming irrelevant for the triggering process.

\section{(g) Variation with input pulse magnitude at a lower linear} growth rate $\gamma=50 \mathrm{~dB} / \mathrm{s}$

Revisiting Fig. 9, we see that even very weak input pulses $(\sim 0.01 \mathrm{pT})$ in the simulation code trigger emissions. Such a situation begins to resemble that of absolute instability, where any signal whatsoever will trigger. In order to be closer to experimentally observed behaviour we now take the base case $(60 \mathrm{~dB} / \mathrm{s})$ but with a weaker linear growth rate of $50 \mathrm{~dB} / \mathrm{s}$, and vary the input pulse magnitude again. The result, shown in Fig. 15, concurs better with reality. For input amplitudes above $0.08 \mathrm{pT}$, strong self sustaining risers are produced with sweep rates $\sim 900 \mathrm{~Hz} / \mathrm{s}$ and with little dependence on the initial amplitude. Below $0.08 \mathrm{pT}$ only weak non self sustaining features are triggered, with one curious exception. With an input amplitude of $0.03 \mathrm{pT}$ a good riser finally appeared but after a substantial delay. It thus appears that the triggering process has an element 
of randomness and chaos, so that it is never possible to predict exactly what will result. The reason for the weak triggering at low input amplitudes is that the input pulse is not magnified to fully nonlinear levels by the time that it reaches the equator. The pulse 'escapes' downstream before a strongly nonlinear, self sustaining riser GR can be established.

(h) Variation with input pulse length at a linear growth rate of $\gamma=50 \mathrm{~dB} / \mathrm{s}$

We now repeat the variation of input pulse length at the lower linear growth rate of $50 \mathrm{~dB} / \mathrm{s}$, and present the results in Fig. 16. At this lower growth rate it becomes harder to trigger strong emissions. With input pulse lengths less than $200 \mathrm{~ms}$, triggering occurs in a rather unpredictable way. Below input lengths of $100 \mathrm{~ms}$ only weak features result, usually fallers with no strong self sustaining emission. Again, at pulse lengths over $200 \mathrm{~ms}$ predictable risers result, with sweep rates as high as $1450 \mathrm{~Hz} / \mathrm{s}$.

\section{Conclusions}

In this work we have used a well established 1-D Vlasov Hybrid Simulation numerical code for the simulation of triggered VLF emissions. We have systematically varied each input parameter in turn, in order to investigate how emission triggering depends on individual simulation parameters and to understand what factors determine the emission sweep rate and spectral form - riser, faller or hook. Figures 8-16 present these results.

It has become clear that, in order for triggering of strong emissions to occur, particle trapping by the wave must occur by the time that the triggering pulse reaches the equator. For this to happen, both the initial pulse amplitude and pulse length must be above minimum values. At a moderate linear growth rate at the equator of $50 \mathrm{~dB} / \mathrm{s}$ these triggering thresholds were clearly apparent. However, at the higher growth rate of $60 \mathrm{~dB} / \mathrm{s}$ the manner of behaviour resembled far more an absolute instability when almost any input will trigger an emission.

Overall, we have noted a tendency to trigger risers preferentially, particularly at intermediate growth rates. This confirms the Siple experimental studies. At least as far as this code goes, fallers tend to be produced at higher growth rates, $>100 \mathrm{~dB} / \mathrm{s}$, and where the linear growth rate is a monotonically increasing function of frequency the code often produces hooks or oscillating tones. In situations in which power input into the wavefield is weak, namely a low linear growth rate, small input amplitude, short input pulse, or low saturation amplitude, the code tends to produce low amplitude fallers, of short duration, often triggered from the end of the input pulse. Again this confirms the experimental studies of Siple triggered emissions.

The question of what determines the value of frequency sweep rate has long been a puzzle. Clearly, and somewhat surprisingly, the linear growth rate and the saturation

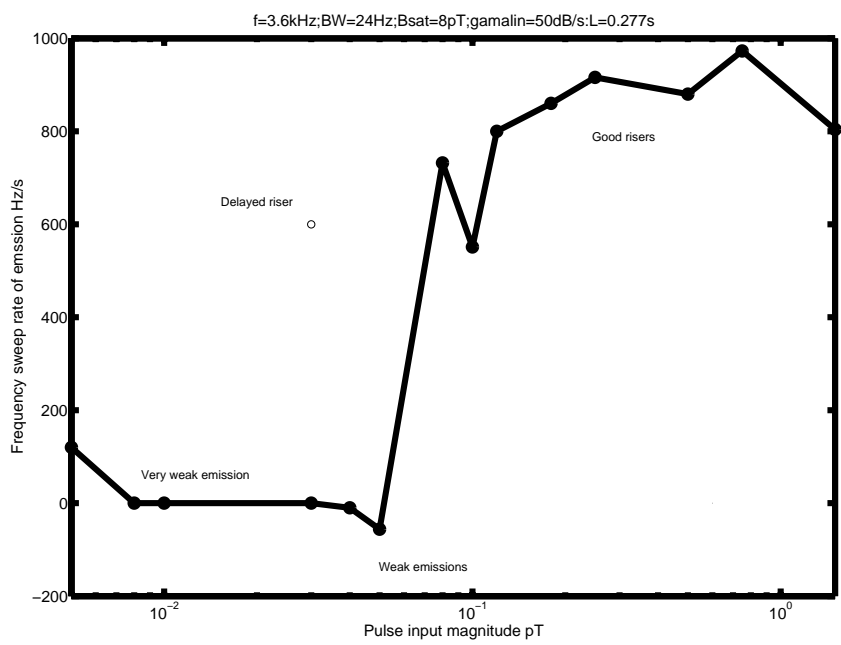

Fig. 15. Dependence of the sweep rate on the input pulse magnitude for a linear growth rate of $50 \mathrm{~dB} / \mathrm{s}$, which is weaker than for the base case. Now the amplitude threshold is more pronounced, and below about $0.08 \mathrm{pT}$ strong triggering does not occur because nonlinear amplitudes are not attained by the time that the pulse reaches the equator.

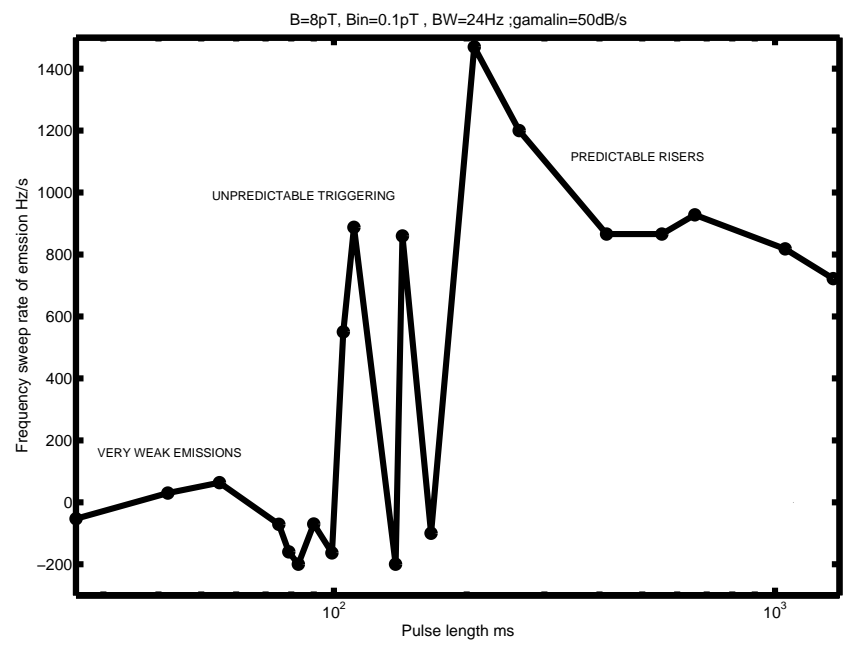

Fig. 16. Dependence of the sweep rate on the pulse length with a weaker linear growth rate of $50 \mathrm{~dB} / \mathrm{s}$. Stable triggering only occurs when the pulse length exceeds $200 \mathrm{~ms}$ in this case.

amplitude do not have much influence on the sweep rate. At a cold plasma density of $400 / \mathrm{cc}$ the riser sweep rate is invariably $\sim 1 \mathrm{kHz} / \mathrm{s}$ and the faller sweep rate $\sim-600 \mathrm{~Hz} / \mathrm{s}$. This study points to the fact that it is the value of the cold plasma density in the equatorial plane that mainly controls the sweep rate magnitude. The sweep rates become much larger as the density drops.

The code itself has two parameters that are system parameters and which have nothing to do with experimental reality. These are the code bandwidth and the saturation amplitude. Fortunately, perhaps, it transpires that, provided that the bandwidth is above about one half the highest trapping 
frequency and the saturation amplitude is well into the trapping regime $(>4 \mathrm{pT})$, the emission triggering behaviour is largely independent of these parameters.

This parameter study has produced some definite predictions regarding triggering behaviour, as outlined above. It would be interesting to perform a corresponding parameter study for the real data to see if these predictions are borne out. As far as ground based data are concerned the Sodankyla Geophysical Observatory, the British Antarctic Survey's base at Halley Bay and Stanford's Siple experiment all have produced large data bases suitable for this task. Of course parameters such as the cold plasma density are best observed on a satellite, and space missions such as Cluster (Santolik et al., 2003) provide the best source for a detailed experimental parametric study of VLF emissions.

Regarding future theoretical developments the aim must be to progress towards developing a 2-D code to model self consistent nonlinear wave particle interactions for a wavefield ducted in a slab geometry. This should verify whether nonlinear unducting loss can act as a saturation mechanism or not. Also as more computational power becomes available one dimensional runs need to be made with higher bandwidth and more numerical precision. One aspect of the triggered emission problem remains unsolved. This is the slow exponential rise of received amplitude when the incoming signal is low amplitude and key down. This is still something of a mystery and demands further ongoing research. Another problem area is the generation of electrostatic waves as a result of the nonlinear electron cyclotron interaction, and whether the consequent diffusive feedback to the zero order distribution function will be significant, possibly as a saturation mechanism also.

Acknowledgements. The authors gratefully acknowledge financial support from NATO Linkage grant PST-CLG-980041 and from INTAS contract 03-51-4132.

Topical Editor T. Pulkkinen thanks R. L. Dowden and another referee for their help in evaluating this paper.

\section{References}

Carlson, C. R., Helliwell, R. A., and Inan, U. S.: Space time evolution of whistler mode wave growth in the magnetosphere, J. Geophys. Res., 95, 15 073-15 089, 1990.

Cheng, C. Z. and Knorr, G.: The integration of the Vlasov equation in configuration Space, J. Comput. Physics, 22, 330-335, 1976.

Helliwell, R. A. and Katsufrakis, J. P.: VLF wave injection into the magnetosphere from the Siple station, Antarctica, J. Geophys. Res., 79, 2511-2518, 1974.

Helliwell, R. A., Carpenter, D. L., and Miller, T. R.: Power threshold for growth of coherent VLF signals in the magnetosphere, J. Geophys. Res., 85, 3360-3366, 1980.
Helliwell, R. A., Carpenter, D. L., Inan, U. S., and Katsufrakis, J. P.: Generation of band limited VLF noise using the Siple transmitter: a model for magnetospheric hiss, J. Geophys. Res., 91, 4381-4392, 1986.

Helliwell, R. A.: Controlled stimulation of VLF emissions from Siple station, Antarctica, Radio Sci., 18, 801-814, 1983.

Helliwell, R. A.: VLF stimulated experiments in the magnetosphere from Siple station Antarctica, Revs. Geophysics, 26, 551-578, 1988.

Helliwell, R. A.: Triggering of whistler mode emissions by the band limited impulse (BLI) associated with amplified VLF emissions from Siple station Antarctica, Geophys. Res. Lett., 27\#10, 14551458, 2000.

Nunn, D.: A self consistent theory of triggered VLF emissions, Planet. Space Sci., 22, 349-378, 1974.

Nunn, D., Demekhov, A., Trakhtengerts, V., and Rycroft, M. J.: VLF emission triggering by a highly anisotropic electron plasma, Ann. Geophys., 21, 481-492, 2003,

SRef-ID: 1432-0576/ag/2003-21-481.

Nunn, D.: The Vlasov Hybrid Simulation Technique - an efficient and stable algorithm for the numerical simulation of collisionfree plasma, Transport Theory and Statistical Physics, vol 34, no1, 151-171, 2005.

Nunn, D., Omura, Y., Matsumoto, H., Nagano, I., and Yagitani, S.: The numerical simulation of VLF chorus and discrete emissions observed on the Geotail satellite, J. Geophys. Res., 102, A12, 27 083-27 097,1997.

Nunn, D., Manninen J., Turunen, T, Trakhtengerts, V. Y., and Erokhin, N.: On the nonlinear triggering of VLF emissions by power line harmonic radiation, Ann. Geophys., 17, 79-94, 1999, SRef-ID: 1432-0576/ag/1999-17-79.

Nunn, D. and Smith, A. J.: Numerical simulation of whistlertriggered VLF emissions observed in Antarctica, J. Geophys. Res., 101, A3, 5261-5277, 1996.

Nunn, D.: A Novel method for the numerical simulation of hot collision free plasmas; Vlasov Hybrid Simulation, J. Comput. Physics, 108, 180-196, 1993.

Nunn, D.: The numerical simulation of nonlinear VLF wave particle interactions using the Vlasov Hybrid Simulation technique, Computer Physics Comms., 60, 1-25, 1990.

Nunn, D.: A nonlinear theory of sideband stability of ducted whistler mode waves, Planetary and Space Science, 34(5), 429451, 1986.

Omura, Y., Nunn, D., Matsumoto, H., and Rycroft, M. J.: A review of observational, theoretical and numerical studies of VLF triggered emissions, J. Atmos. Terr. Physics, 53, 351-368, 1991.

Rycroft, M. J.: Gyroresonance interactions in the outer plasmasphere, J. Atmos. Terr. Physics, 38, 1211-1214, 1976.

Santolik, O., Gurnett, D. A., and Pickett, J. S.: Spatio-temporal structure of storm-time chorus, J. Geophys. Res., 108, A7, 278, doi:10.1029/2002JA009791, 2003.

Smith, A. J. and Nunn, D.: Numerical simulation of VLF risers, fallers and hooks observed in Antarctica, J. Geophys. Res., 103, A4, 6771-6784, 1997.

Trakhtengerts, V. Y. and Rycroft, M. J.: Whistler electron interactions in the magnetosphere: new results and novel approaches, J. Atmos. Sol.-Terr. Physics, 62, 1719-1733, 2000. 